\title{
Vibrio parahaemolyticus
}

National Cancer Institute

\section{Source}

National Cancer Institute. Vibrio parahaemolyticus. NCI Thesaurus. Code C86846.

A species of facultatively anaerobic, Gram negative, curved rod shaped bacteria assigned to the phylum Proteobacteria. This species is motile, oxidase and lysine decarboxylase positive, hemolytic, halophilic and can use a wide variety of sugars as carbon sources. V. parahaemolyticus is a marine organism and a pathogen that causes gastroenteritis and wound infections. 